\section{Clinical significance of intraabdominal pressure and abdominal perfusion pressure in patients with acute abdominal syndrome}

\author{
NATAŠA KOVAČ • MLADEN ŠIRANOVIĆ • BRANKA MAZUL-SUNKO
}

\author{
NATAŠA KOVAČ $(\bowtie) \bullet$ \\ MLADEN ŠIRANOVIĆ • \\ BRANKA MAZUL-SUNKO \\ Department of Anaesthesiology \\ and Intensive Care, \\ University Hospital \\ "Sestre Milosrdnice", \\ Vinogradska 29 \\ 10000 Zagreb, Croatia \\ Phone: 38513787575 \\ Fax: 38513787796 \\ E-mail: nkovac@inet.hr
}

\begin{abstract}
Elevated intraabdominal pressure (IAP) has been claimed to play a role in abdominal compartment syndrome. We assessed the correlation between the values of IAP, abdominal perfusion pressure (APP) and clinical scoring systems including SIRS, MODS and APACHE II and the patients' survival rate in patients admitted to the ICU with acute abdomen due to ileus, intestinal perforation, peritonitis and trauma.

We measured IAP and APP in 50 surgical patients. In this study the IAP was measured in a non-invasive manner via urinary bladder pressure. The APP was calculated as the difference between mean arterial pressure and IAP values.

A significantly higher IAP was found in the non-survivors' group in comparison with the survivors' group. On the other hand, the APP inversely correlated with disease severity scores including SIRS, MODS and APACHE II, whereas IAP values did not show any correlation to these clinical parameters. These findings suggest that IAP and APP may be useful tools in the clinical evaluation of patients with acute abdomen.
\end{abstract}

Key words: intraabdominal pressure, abdominal perfusion pressure, acute abdominal syndrome

\section{Introduction}

High intraabdominal pressure (IAP) occurs frequently in patients with acute abdominal syndromes such as ileus, intestinal perforation, peritonitis, acute pancreatitis or trauma. An elevated IAP may lead to intraabdominal hypertension (IAH) and abdominal compartment syndrome (ACS). Both $\mathrm{IAH}$ and $\mathrm{ACS}$ are etiologically related to an increased morbidity and mortality of critically ill patients.

The normal value of IAP in surgical patients is between 2 to $10 \mathrm{mmHg}$, the value above $15 \mathrm{mmHg}$ is intraabdominal hypertension and the value above 25 $\mathrm{mmHg}$ is considered an indicator of ACS which leads to organ failure. (1) ACS develops due to an increase in intraabdominal volume. Excessive intraabdominalvolumeincrease exceeds the elastic limits and compensatory extension of the abdominal wall. Normally, the abdominal contents are relatively noncompressive and mainly fluid in its nature.

Therefore, the pressure values follow the hydrostatic laws of Pascal. The gold standard for invasive IAP monitoring is via direct needle puncture; the noninvasive standard is an indirect method via the bladder. (2)

The increase of the volume within the elastic structure of the abdominal wall causes an increase of overall pressure in the cavity and organs and it may decrease tissue blood perfusion.

An increase in abdominal pressure may lead to distant effects in other parts of the body, such as increased intracranial pressure, pericardial tamponade, tension pneumothorax or extremity compartment syndrome. (3) All these elements need to be considered in ACS diagnostic evaluation of the patient.

During ACS the high IAP can lead to cardiovascular failure or dysfunction due to diminished venous return. This is defined via the

the need for vasopressors. Respiratory failure is defined as a $\mathrm{PaO}_{2} / \mathrm{FiO}_{2}$ ratio < 200 with the need for respiratory support in the form of mechanical ventilation. 
Also, elevated IAP can lead to acute renal failure, splanchnic hypoperfusion and gut ischemia. Even remote effects on the brain, such as an increase in intra-cranial pressure, may be caused by high IAP. $(4,8,9)$

Increased risk factors for IAH/ACSdevelopment are conditions following abdominal surgery, major trauma/ burns, gastroparesis, ileus, colonic pseudo-obstruction, hemoperitoneum, pneumoperitoneum, ascites, massivefluid resuscitation and sepsis. (5)

Pathophysiologic effects of elevated IAP in animal models are release of cytokines IL-1b, TNF $\alpha$ and IL-6. The concentration of lysine-vasopressin is increased, formation of oxygen free radicals and decreased cellular production of adenosine triphosphate may be a contributing component. Gut barrier dysfunction and intestinal wall oedema develop, which may contribute to bacterial translocation and endotoxemia. These alternations would lead to multiorgan dysfunction syndrome (MODS) and multiorgan failure (MOF). (6)

\section{Methods}

This prospective study was conducted in 6 months period (from January 2003 to July 2003) in surgical ICU of a teaching hospital.

The 50 surgical patients, age from 18 to 75 , with acute abdomen syndrome were randomly selected because of peritonitis-17patients, obstructivebowel18 patients, pancreatitis- 5 patients and abdominal trauma-10 patients. We measured IAP and calculated APP $(\mathrm{APP}=\mathrm{MAP}-\mathrm{IAP})$ and correlated to the survival and clinical severity scores. They all were scored with SIRS, MODS and APACHE II. The IAP was measured in a non-invasive manner via the urinary bladder filled up with $100 \mathrm{ml}$ of normal saline through the previously drained and clamped standard urinary catheter. (7) The actual pressure is tranduced to a manometer in the mid-axillary line. We analysed the IAP, APP and disease severity scores which were measured upon admission to the ICU.

Statistics: the non-parametric KruskalWallis test is used to compare median values of IAP, MODS and APACHE II of survivors and nonsurvivors, and linear regression was used to compare APP and IAP with SIRS, MODS and APACHE II scores. Both tests are applied in similar clinical Investigations, and linear regression was performed to identify independent predictors.

\section{Results}

In order to evaluated the contribution of IAP to the clinical parameters we compared survivors vs. non-survivors with respect to IAP values. Twenty-five patients who survived had lower IAP than non-survivors.

Figure 1 shows IAP values in the respective groups. The mean IAP amongst the survivors was $15 \mathrm{mmHg}$. It is significantly lower than the values of non-survivors according to the nonparametric statistical Kruscal-Wallis test. The median for non-survivors was $18 \mathrm{mmHg}$ (rang 6-30) and for survivors the median was $15 \mathrm{mmHg}$ (rang 6-36) with significance $p<0.009$.

Expectedly, when survivors were compared with non-survivors according to the disease severity score the significant difference was found for APACHE II and MODS values. In the survivors' group the MODS was 1 organ in dysfunction (rang 0-6) and for nonsurvivors 3 organs in dysfunction (rang

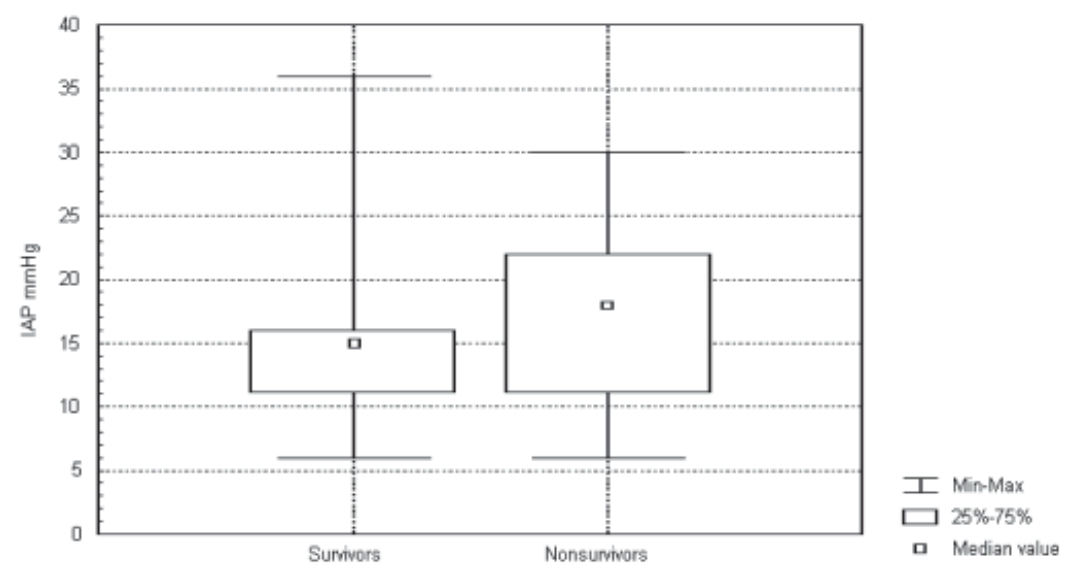

Figure 1. Distribution of IAP (intraabdominal pressure) values for survivors and non-survivors

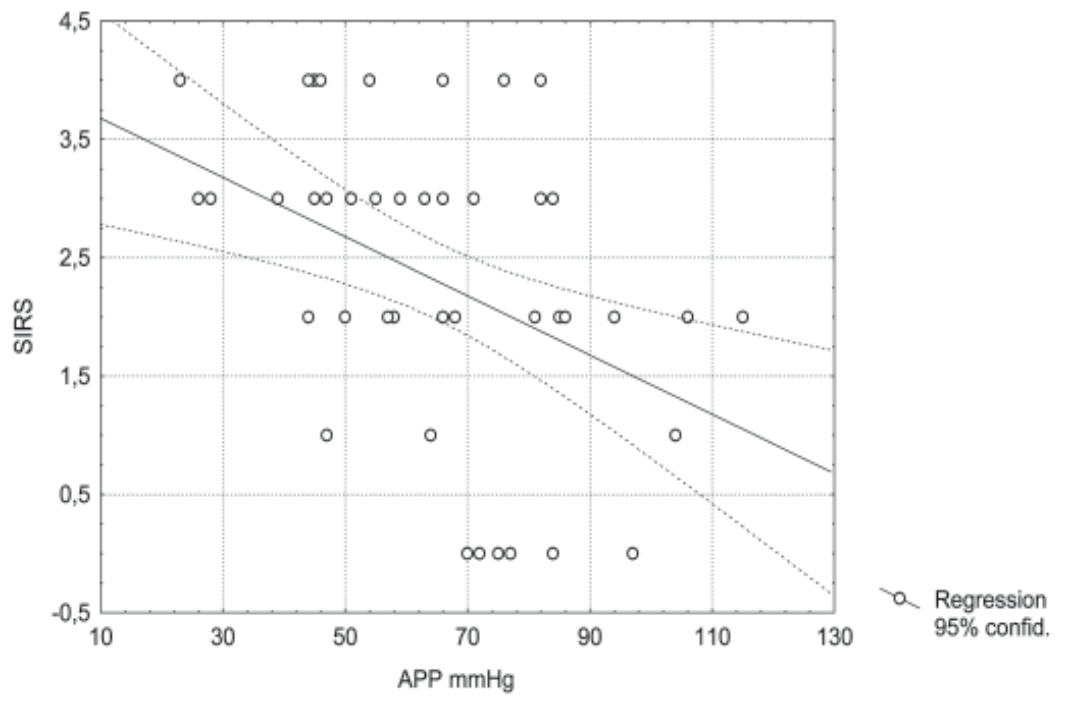

Figure 2. Correlation between APP and SIRS, (APP=Abdominal Perfusion Pressure, SIRS=Systemic Inflammatory Response Syndrome) 


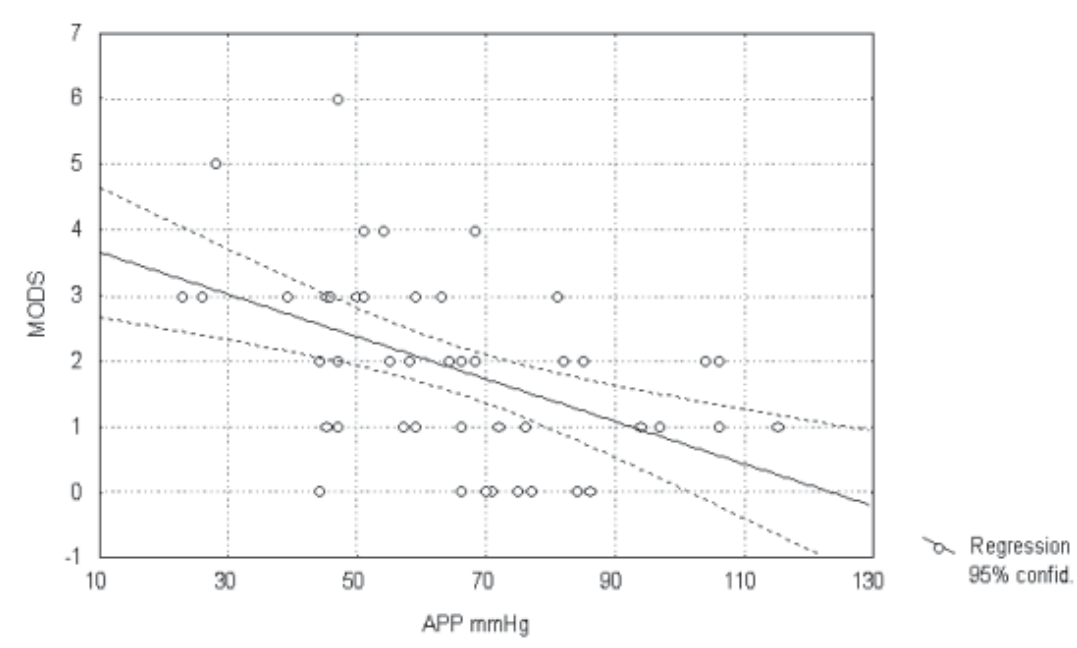

Figure 3. Correlation between APP and MODS (APP =Abdominal Perfusion Pressure, MODS = Multiple Organ Dysfunction Syndrome)

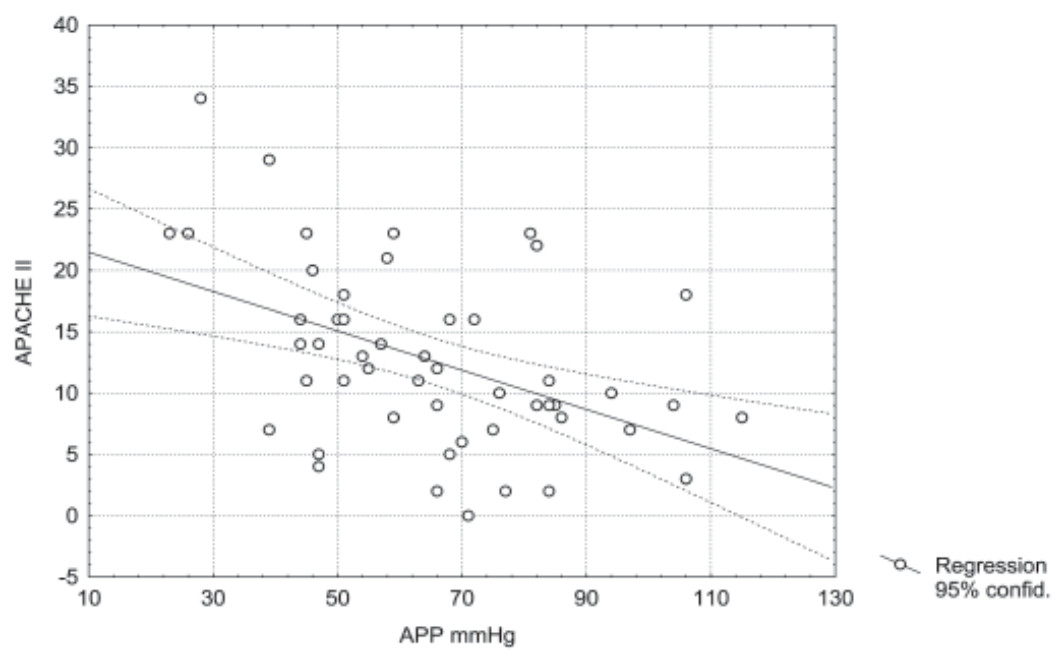

Figure 4. Correlation between APP and APACHE II (APP =Abdominal Perfusion Pressure, APACHE II=Acute Physiology And Chronic Health Evaluation)

$0-6), p<0.0072$. For non-survivors the median APACHE II score was 18 (rang 0-34), and for survivors 9 (rang 2-23), $\mathrm{p}<0.0067$.

Nevertheless when the IAP values were correlated to the disease severity scores no significant relationship was found. On the other hand, linear regression correlation was demonstrated for the comparison of APP with disease severity scores, as follows: APP vs. figure 4 shows lower abdominal perfusion pressure in more severely ill patients respectively withhigher APACHE II score.

Despite the absence of significant correlation between IAP and clinical severity scores, the APP negative contribution to the clinical picture appears to be well established.

\section{Discussion}

This study shows that IAP might have a predictive and prognostic value in surgical patients. From the clinical point of view diagnosis of IAH/ACS is predicatively and prognostic relevant. Ethiopathogenesis of IAP in the establishment of positive feedback loops may worsen intraabdominal and extraabdominal organ dysfunction. Simple clinical measurement of intravesical pressure gives a close estimate of IAP and is a readily available clinical parameter.

In this paper we have shown that the APP may be the better indicator of disease severity.

IAP may influence hemodynamic pressures and thereby may affect organ perfusion. In order to estimate such theoretical contributions we calculated the APP in all 50 patients and correlated this value with disease severity scores. The APP is a good indicator of organ perfusion and these findings corroborate the importance of maintaining appropriate organ perfusion in critically ill patients.

These parameters may be useful tools in the clinical evaluation of patients with an acute abdomen in the ICU. We would like to recommend IAPmeasurement for standard routine usage. Surgical intervention and optimal timing of abdominal cavity decompression based on these parameters may be beneficial to ACS patient. However, a prospective clinical study of outcomes, morbidity and correlations to the dynamics of disease condition are required. 


\section{REFERENCES}

1. Malbrain MLNG, Chiumello D, Pelosi P, Wilmer A, Brienza N, Malcangi V et al. Prevalence of intra-abdominal hypertension in critically ill patients: a multicentre epidemiological study. Intensive Care Medicine 2004;30:822- 9

2. Malbrain MLNG, Jones F. Intra-abdominal pressure measurement Techniques. In: Ivatury RR, Cheatham ML, Malbrain MLNG, Spugrue M, editors. Abdominal Compartment Syndrome. Landes Bioscience; 2006. pp. 19-20.

3. Balogh Z, Moore FA. Recent advances in the characterisation of post injury abdominal syndrome. Inter J of Intensive Care 2004;11(1):30-42.

4. Malbrain MLNG, Deeren D, De Potter TJR. Intra-abdominal hypertension in the critically ill: it is time to pay attention. Curr Opin Crit Care 2005;11:156-171.

5. Walker J, Criddle LM. Pathophysiology and management of abdominal compartment syndrome. American Journal of Critical Care 2003 Jul;12(4): 367-371.

6. World Society of the Abdominal Compartment Syndrome (WSACS). Intra-abdominal Hypertension (IAH) assessment algorithm ŠonlineĆ. 2007 July 7 Šcited 2007 July 20Ć; Available from: URL:http://www.wsacs.org/

7. Kron IL, Harman, Nolan SP. The measurement of intraabdominal pressure as a criterion for abdominal re-exploration. Ann Surg 1984; 199:28-30.

8. Malbrain MLNG. Is it wise not to think about intraabdominal hypertension in the ICU. Curr Opin Crit Care 2004;10:132-45.

9. De Waele JJ, Hoste EAJ, Malbrain MLNG. Decompressive laparotomy for abdominal compartment syndrome-a critical analysis. 